\title{
Therapeutic potential of RNA interference in neurodegenerative diseases
}

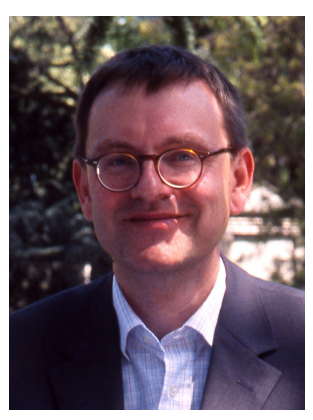

Alexander Pfeifer Institute of Pharmacology \& Toxicology, University of Bonn, Reuterstr. 2B, Germany

Tel.: +49892180 77654; Fax: +49892180 77326; alexander.pfeifer@ uni-bonn.de

\author{
'RNAi is a potent tool to \\ suppress specific genes in \\ human cells and has great \\ potential for therapeutic use in \\ neurological disorders.'
}

RNA interference (RNAi) is a cellular process that eventually leads to the degradation of mRNAs and silencing of gene expression (for detailed reviews see [1-3]). This powerful mechanism of gene silencing at a post-transcriptional level has been identified in a wide spectrum of eukaryotes, including plants, yeast, worms, flies and mammals. The signals that trigger RNAi are small double-stranded (ds)RNA molecules. RNAi-mediated gene silencing is a step-wise biochemical process: the dsRNAs are converted by the cellular enzyme Dicer, to socalled short interfering RNAs (siRNAs). The siRNAs are incorporated into the RNA-induced silencing complex (RISC) and guide the RISC to complementary mRNA molecules, thereby triggering gene-specific mRNA degradation.

Small dsRNAs can be generated during infection with RNA viruses. Another important class of RNAi triggers are short hairpin RNAs (shRNAs) and micro RNAs (miRNAs) that form stem-loop structures. miRNAs are generated from longer RNA molecules that are encoded in the genome of worms, flies and mammals. Mature miRNAs bind to the 3 ' untranslated region of mRNAs and suppress translation of the bound mRNA. In addition, miRNAs have been shown to induce degradation of the target mRNA molecule. To date, more than 500 miRNAs have been identified and approximately $30 \%$ of human genes have been postulated to be regulated by miRNAs.

RNAi is a potent tool to suppress specific genes in human cells and has great potential for therapeutic use in neurological disorders. Exogenouslyproduced siRNA molecules can be directly used to silence cellular gene expression. However, this requires chemical or enzymatic synthesis, as well as efficient RNA transfer to the target cells (e.g., by RNA transfection or electroporation) [4]. An alternative approach is to produce siRNAs or shRNAs within the target cell from RNA polymerase III transcription units. Presently, the most widespread siRNA/shRNA-expression cassettes contain either the promoter of RNAse P, RNA H1, or small nuclear RNA U6. Transfer of these expression cassettes into neuronal cells can be efficiently achieved by viral vectors in vitro and in vivo. In this editorial, I will focus on viral vectors for the induction of RNAi.

\section{Viral vectors for the induction of RNA interference in the CNS}

Given the present lack of effective pharmacological therapies for neurodegenerative diseases, genetherapy approaches are a promising alternative to classical pharmacology. At present, the most widely used viral vectors for gene transfer in the nervous system are adeno-associated virus (AAV) and lentiviral vectors. In addition, vectors derived from herpes viruses also efficiently transduce neurons (for a review of viral vectors see $[5,6]$ ).

\section{'...approximately $30 \%$ of human genes \\ have been postulated to be regulated by miRNAs.'}

AAVs are parvoviruses that contain a $4.7 \mathrm{~kb}$ DNA genome. AAVs can establish a latent state of infection through integration into a specific region of human chromosome 19 [7]. This important feature of AAV is dependent on the action/presence of the AAV protein Rep, which is normally deleted from AAV vectors. However, $A A V$ vectors do not require integration for gene expression and most of the transgene expression from these vectors results from extrachromosomal vectors that persist as episomes.

$A A V$ vectors have been extensively used for gene delivery vehicles in the nervous system as they target primarily neurons and result in sustained gene expression [8]. In addition, AAV vectors derived from different serotypes display differential efficacy and tropism, suggesting that vectors based on distinct AAV serotypes can be chosen for specific applications in the nervous system.

Lentiviral vectors are attractive tools for gene transfer in the nervous system. Lentiviruses belong to the large family of retroviruses. The most widely used lentivirus-derived vector systems are derived from HIV [9]. In contrast with vectors derived 
from prototypic retroviruses (e.g., murine leukemia virus), lentiviral vectors do not require cell division for transduction and, therefore, efficiently transduce nondividing cells such as neurons.

From a practical point of view, the major differences between $\mathrm{AAVs}$ and lentiviruses are:

- Lentiviral vectors have a significantly larger packaging capacity

- Lentivectors efficiently integrate into the host genome

- Integration is not random, but HIV-derived vectors clearly favor transcription units [10]

- Like all retroviruses, lentiviruses are enveloped viruses

The tropism of lentiviruses can be changed through the incorporation of envelope proteins of other viruses into the viral particles (pseudotyping). Lentivectors are most commonly pseudotyped with the vesicular stomatitis virus envelope glycoproteins (VSV-G) [6]. Experimental data of several groups [9,11,12] clearly demonstrate that neurons, as well as glial cells, are efficiently transduced by VSV-G pseudotyped lentivectors in vivo. AAV vectors can be pseudotyped with capsids of other serotypes by, for example, packaging of $\mathrm{AAV}-2$ genomes into AAV-6 or -8 capsids (crosspackaging).

\section{'...the high frequency of antibodies against AAV ... could be a major limitation for gene therapy using these vectors.'}

At present, approximately 4 and $1 \%$ of all clinical gene-therapy trials are based on AAV and lentiviral vectors, respectively [101]. Although no pathology is known to be associated with AAV, the high frequency of antibodies against AAV ( $80 \%$ of the human population are seropositive for the most common AAV subtype) could be a major limitation for gene therapy using these vectors. On the other hand, there are important safety issues with lentivectors (e.g., insertional mutagenesis) that have to be addressed.

Taken together, the combination of viral vectors with RNAi has great potential for modulating the expression of genes involved in neurodegenerative disorders.

\section{Animal models for RNA interference}

RNAi-based therapeutic approaches have been developed for several neurodegenerative disorders [13,14]. Among others, siRNA applications have been developed for polyglutamine disorders.
These disorders are caused by dominantly-inherited genes that carry abnormally extended glutamine (CAG) repeats (e.g., Huntington's disease and cerebellar ataxias).

\section{'...the combination of viral vectors with RNAi has great potential for modulating the expression of genes involved in neurodegenerative disorders.'}

The logical aim of siRNA-based strategies is to target the pathological repeats [15]. However, this approach is hampered by the fact that the wildtype gene could also be affected by the siRNAs. Amyotrophic lateral sclerosis (ALS) is another example for which siRNA therapies have been developed. Two groups achieved a considerable delay in the onset of ALS symptoms and even an extension in survival in an ALS mouse model using lentiviral siRNA vectors [16,17]. Alzheimer's disease $(\mathrm{AD})$ is the most common neurodegenerative disease, and the therapeutic potential of siRNA has been demonstrated for transgenic AD mouse models [18]. However, the widespread neuropathology of $\mathrm{AD}$ in humans will be a major hurdle for the successful use of siRNAs. The rather localized neuropathology of Parkinson's disease (PD) make this neurodegenerative disease a good candidate for RNAi therapies. However, the lack of animal models that faithfully mimic human PD pathologies, as well as the plethora of possible target genes, hamper the development of siRNA-based strategies for PD.

RNAi also has great potential for the treatment of 'unusual' neurodegenerative diseases, such as prion diseases (transmissible spongiforme encephalopathies [TSEs]). According to the protein-only hypothesis, prion diseases are caused by an untypical infectious agent; the disease-specific isoform of the host-encoded cellular prion protein $(\mathrm{PrPC})$. The infectious agent $\left(\mathrm{PrP}^{\mathrm{Sc}}\right.$ or $\left.\mathrm{Pr}^{\mathrm{Pres}}\right)$ is protease resistant, forms aggregates and accumulates in the infected cells [19]. To date, the vast majority of antiinfectious siRNA approaches directly target the infectious agent. However, targeting host genes involved in infectious disease - for example, genes required for propagation of the infectious agent is a promising alternative approach. Accordingly, several groups developed siRNAs that target $\mathrm{PrP}^{\mathrm{C}}$ [20-22]. Importantly, targeting of $\mathrm{PrPC}^{\mathrm{C}}$ by lentivirally delivered shRNAs reduces $\mathrm{PrPSc}$ accumulation and prolongs survival in a mouse model of TSE [22], clearly demonstrating the potential of RNAi for treatment of TSEs. 


\section{Conclusions}

Although siRNA technology has had great success in the laboratory, its application in the clinic requires that the major issues are addressed, including:

- Optimized siRNA design - it is critical to identify siRNAs that achieve an efficient and selective knockdown of the target gene. Although several parameters for siRNA design have been identified that should provide efficient induction of RNAi, the efficacies of siRNAs can only be roughly estimated in silico at present;

- Transduction efficacy - lentiviral and AAV vectors are highly advanced and widely used tools for gene transfer. High transduction efficacies for neuronal cells in vivo can be reproducibly achieved by these vectors;

- Delivery of the vectors - efficient delivery of siRNA vectors is central to their use for human therapy. Delivery of the viral siRNA vectors to the brain is by no means trivial. Single stereotactical injections will allow only a limited number of CNS regions to be targeted. Multiple injections of the brain could provide a means for a more widespread delivery of siRNA vectors. Catheter placement has been developed for more widespread and constant delivery of synthetic siRNAs. This could also be a route to deliver viral siRNA to larger brain areas. The close interaction of scientists from different fields of life sciences - including neuroscience, virology, molecular pharmacology, biotechnology and others - will be necessary to overcome this issue;

- Safety issues - immunological response (e.g., against AAV) and insertional mutagenesis (of retroviral vectors) are only two examples of safety issues that have to be addressed before the use of viral siRNA vectors in humans. In addition, there are also safety issues concerning the siRNA itself. siRNAs can have nonspecific effects, such as off-target silencing and activation of the interferon pathway. In addition, oversaturation of the miRNA/shRNA pathway after delivery of multiple high-dose AAV siRNA vectors has been observed in mice [23].

\section{Future perspective}

Clinically relevant animal models are essential for the future development of siRNA therapies. The progress in transgenic technology - especially lentiviral transgenesis [24] - will further the development of much needed large-animal models.

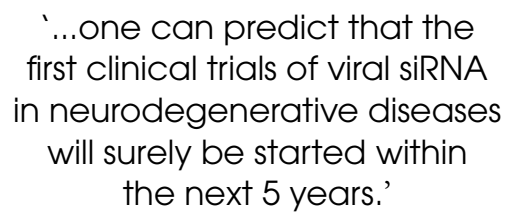

In addition, future research will have to focus on the technical issues at hand, especially efficient delivery of siRNAs and/or siRNA vectors into the CNS. It will be of utmost importance to achieve widespread transfer of synthetic siRNAs and siRNA vectors in the CNS. On the other hand, targeting specific brain areas will be necessary for neurodegenerative diseases such as PD. The blood-brain barrier constitutes a physical barrier for both synthetic siRNAs as well as siRNA vectors. Given the success of siRNAs in rodent models, one can predict that the first clinical trials of viral siRNA in neurodegenerative diseases will surely be started within the next 5 years.

\section{Executive summary}

\section{Introduction}

- RNA interference has great potential for the treatment of neurodegenerative disorders.

Viral vectors for the induction of RNA interference in the CNS

- Adeno-associated virus and lentiviral vectors efficiently deliver small interfering (si)RNAs to neuronal cells.

\section{Animal models for RNA interference}

- A broad spectrum of siRNA interventions have been tested in rodent models.

\section{Conclusions}

- RNAi has been successfully used in the laboratory, but, for clinical use, technical issues and safety issues have to be addressed.

\section{Future perspective}

- Future research should focus on the efficient and widespread induction of RNAi in the CNS. Novel, more clinically relevant models are needed for many neurodegenerative disorders to test siRNA therapies. 


\section{Bibliography}

1. Novina CD, Sharp PA: The RNAi revolution. Nature 430, 161-164 (2004).

2. Mello CC, Conte D Jr: Revealing the world of RNA interference. Nature 431, 338-342 (2004).

3. Hannon GJ, Rossi JJ: Unlocking the potential of the human genome with RNA interference. Nature 431, 371-378 (2004).

4. Tuschl T: Expanding small RNA interference. Nat. Biotechnol. 20, 446-448 (2002).

5. Verma IM, Weitzman MD: Gene therapy: twenty-first century medicine. Annu. Rev. Biochem. 74, 711-738 (2005).

6. Pfeifer A, Verma IM: Gene therapy: promises and problems. Annu. Rev. Genomics Hum. Genet. 2, 177-211 (2001).

7. McCarty DM, Young SM Jr, Samulski RJ: Integration of adeno-associated virus (AAV) and recombinant AAV vectors. Annu. Rev. Genet. 38, 819-845 (2004).

8. Mandel RJ, Manfredsson FP, Foust KD et al: Recombinant adeno-associated viral vectors as therapeutic agents to treat neurological disorders. Mol. Ther. 13, 463-483 (2006).

9. Naldini L, Blomer U, Gallay P et al: In vivo gene delivery and stable transduction of nondividing cells by a lentiviral vector. Science 272, 263-267 (1996).

10. Bushman FD: Targeting survival: integration site selection by retroviruses and LTR-retrotransposons. Cell 115, 135-138 (2003).
11. Pfeifer A, Brandon EP, Kootstra N, Gage FH, Verma IM: Delivery of the Cre recombinase by a self-deleting lentiviral vector: efficient gene targeting in vivo. Proc. Natl Acad. Sci. USA 98, 11450-11455 (2001).

12. Jakobsson J, Ericson C, Jansson M, Bjork E, Lundberg C: Targeted transgene expression in rat brain using lentiviral vectors. J. Neurosci. Res. 73, 876-885 (2003).

13. Ralph GS, Mazarakis ND, Azzouz M: Therapeutic gene silencing in neurological disorders, using interfering RNA. J. Mol. Med. 83, 413-419 (2005).

14. Boudreau RL, Davidson BL: RNAi therapy for neurodegenerative diseases. Curr. Top. Dev. Biol. 75, 73-92 (2006).

15. Xia H, Mao Q, Eliason SL et al:: RNAi suppresses polyglutamine-induced neurodegeneration in a model of spinocerebellar ataxia. Nat. Med. 10, 816-820 (2004).

16. Ralph GS, Radcliffe PA, Day DM et al: Silencing mutant SOD1 using RNAi protects against neurodegeneration and extends survival in an ALS model. Nat. Med. 11, 429-433 (2005).

17. Raoul C, Abbas-Terki T, Bensadoun JC et al: Lentiviral-mediated silencing of SOD1 through RNA interference retards disease onset and progression in a mouse model of ALS. Nat. Med. 11, 423-428 (2005).

18. Singer $\mathrm{O}$, Marr RA, Rockenstein $\mathrm{E}$ et al.: Targeting BACE1 with siRNAs ameliorates Alzheimer disease neuropathology in a transgenic model. Nat. Neurosci. 8 , 1343-1349 (2005).
19. Prusiner SB: Novel proteinaceous infectious particles cause scrapie. Science 216, 136-144 (1982).

20. Daude N, Marella M, Chabry J: Specific inhibition of pathological prion protein accumulation by small interfering RNAs. J. Cell Sci. 116, 2775-2779 (2003)

21. Tilly G, Chapuis J, Vilette D, Laude H, Vilotte JL: Efficient and specific downregulation of prion protein expression by RNAi. Biochem. Biophys. Res. Commun. 305, 548-551 (2003).

22. Pfeifer A, Eigenbrod S, Al-Khadra S et al.: Lentivector-mediated RNAi efficiently suppresses prion protein and prolongs survival of scrapie-infected mice. J. Clin. Invest. 116, 3204-3210 (2006).

23. Grimm D, Streetz KL, Jopling CL et al: Fatality in mice due to oversaturation of cellular micro RNA/short hairpin RNA pathways. Nature 441, 537-541 (2006)

24. Pfeifer A: Lentiviral transgenesis. Transgenic Res. 13, 513-522 (2004).

\section{Website}

101. Gene Therapy Clinical Trials Worldwide www.wiley.co.uk/genetherapy/clinical/

\section{Affiliation}

- Alexander Pfeifer Institute of Pharmacology \& Toxicology, University of Bonn, Reuterstr. 2B, Germany Tel.: +49892180 77654; Fax: +49892180 77326; alexander.pfeifer@uni-bonn.de 